$\underline{\text { Case Report }}$

\title{
Primary subcutaneous hydatid cyst in supraclavicular region diagnosed by fine needle aspiration cytology: A rare case report
}

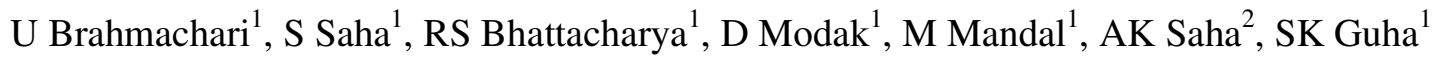 \\ Sri Lankan Journal of Infectious Diseases 2015 Vol.5 (1):32-35 \\ DOI: http://dx.doi.org/10.4038/sljid.v5i1.7613
}

\begin{abstract}
:
Hydatid cyst is not an uncommon disease, primarily affecting the liver, lungs and other viscera. In case of disseminated disease, cysts may be found in uncommon locations like muscle and subcutaneous tissue. However, the presence of a hydatid cyst in subcutaneous tissue only, without being present in liver or lungs is an extremely rare entity. Here we report such a rare case of solitary primary subcutaneous hydatid cyst, misdiagnosed previously as a cold abscess until aspiration clinched the diagnosis.

A 46 year old female patient presented with a gradually progressive swelling in the right supraclavicular region. Imaging studies favoured a cold abscess. Haematoxylin and Eosin stained aspirated fluid from the swelling showed the presence of scolices with hooklets. This finding conclusively proved the swelling to be a hydatid cyst. Serological tests were negative. No other organ involvement was found. The case was finally diagnosed to be an extremely rare case of primary solitary subcutaneous hydatid cyst in the supraclavicular region. The cyst was surgically removed.

This case points to the fact that although hydatid cyst is a rare possibility, it should be considered in the differential diagnosis of any cystic lesion. Without the correct diagnosis, proper therapy can be missed and might lead to recurrence of the disease.
\end{abstract}

Keywords: primary hydatid cyst, subcutaneous, supraclavicular, FNAC

\section{Introduction:}

Hydatid cyst disease is caused by larval tapeworms of Echinococcus species. Amongst them, E. granulosus is the commonest organism to affect humans. Animals like dogs, wolves and foxes are the definitive hosts, while sheep, goats, camels and horses are the usual intermediate hosts of E. granulosus. Humans are inadvertent intermediate hosts for Echinococcus species. Humans acquire echinococcosis by ingesting viable eggs of the parasite. In the intestine, the eggs hatch and release oncospheres, which enter the circulation after penetrating the mucosa. Inside the viscera, the oncospheres encyst and over time, develop to a mature hydatid cyst. The most common location of a hydatid cyst is the liver, followed by lung and other viscera.

\footnotetext{
${ }^{1}$ Department of Tropical Medicine, Calcutta School of Tropical Medicine, Kolkata, West Bengal, India ${ }^{2}$ Department of General Surgery, Nil Ratan Sircar Medical College and Hospital, Kolkata, West Bengal, India Address for correspondence: Dr. Uttiya Brahmachari., Department of Tropical Medicine, Calcutta School of Tropical Medicine, Kolkata, West Bengal, India Email - uttiya@live.in, uttiya.nrs85@gmail.com
} 
Here we report an extremely rare case of primary hydatid cyst located in the subcutaneous region at the side of neck which was diagnosed accidentally by aspiration cytology.

\section{Case Report:}

A 46 year old female patient, residing in Kolkata, attended the outpatient department (OPD) of our hospital with a swelling on the right side of her neck. She noticed the swelling about 2

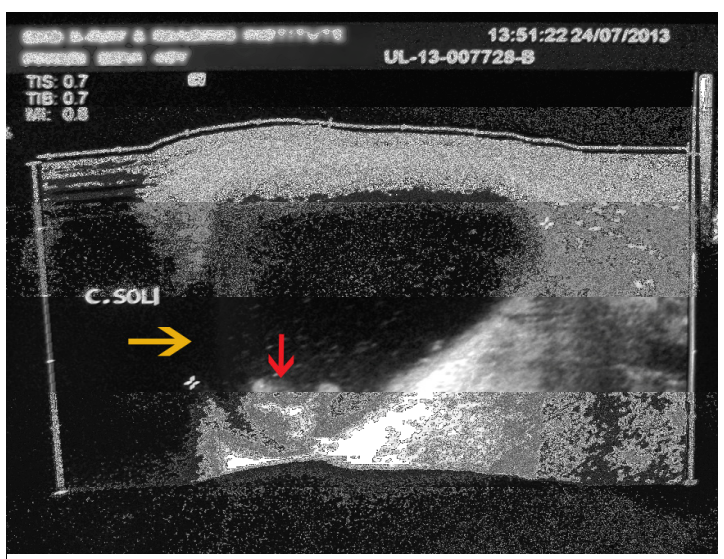

Figure 1: USG of swelling showing a cystic SOL (yellow arrow) with internal echoes (red arrow) months ago when it was the size of a pea. The swelling was gradually increasing in size and at the initial visit was approximately $6 \mathrm{~cm} \times 5 \mathrm{~cm}$ in size. On examination the swelling was superficial, fluctuant, transluminant, non-tender, nonreducible, non-pulsatile and free from skin and deeper structures. An ultra sonogram of the swelling was done, which revealed a cystic swelling of 5.4 $\mathrm{cm} \times 4 \mathrm{~cm}$ size with the presence of internal echogenicity (figure 1).| Doppler study of the swelling showed no vasculature. A provisional diagnosis of cold abscess was made and fine needle

aspiration (FNAC) of the swelling was carried out. The aspirate was a straw coloured, translucent liquid, which was sent for microscopic examination. Zeihl Neelsen stain of the fluid smear showed no acid fast bacilli. Haemotoxylin \& Eosin stain showed multiple pus cells and was reported as an infected lymphatic cyst. The wet mount of the fluid was then examined under high power $(400 \mathrm{x}$ magnification) which showed spherical masses with hooklets, identified as scolices of Echinococcus species. The stained fluid was reexamined under oil immersion field (1000x magnification) and hooklets were identified (figure 2).

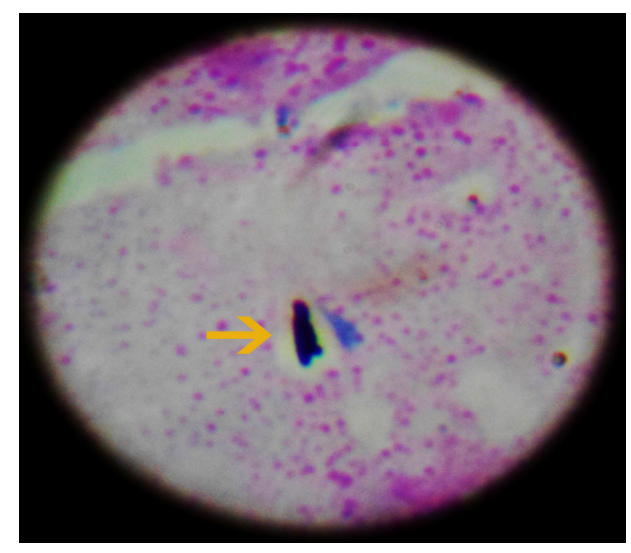

Figure 2. Hooklet seen under microscope (yellow arrow) x1000

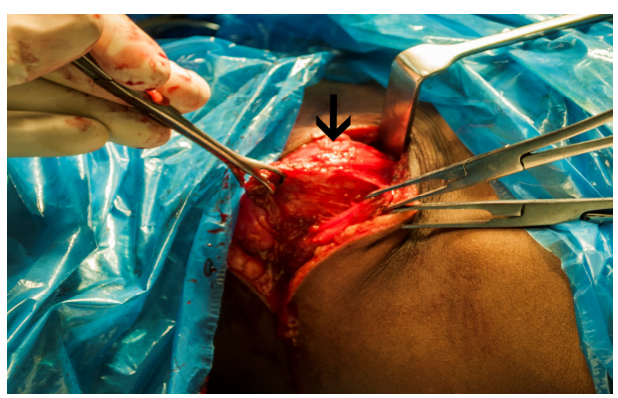

Figure 3. Surgical excision of the hydatid cyst (black arrow)

The patient underwent other tests to find out the primary cyst. Chest skiagram and ultra sonogram of the abdomen was done and no pathology was found. Anti echinococcal antibody assay was negative. A final diagnosis of primary subcutaneous hydatid cyst in the supraclavicular region was made. The patient was treated with oral albendazole followed by surgical excision of the cyst (figure 3 ). 


\section{Discussion:}

Hydatid cyst is a zoonotic disease, with human beings as accidental hosts. This disease is endemic in the Middle East as well as in other parts of the world, including Africa, India, South America, Turkey, and Southern Europe. ${ }^{1}$ In these regions, the incidence ranges from 1220 cases per 100,000 inhabitants. ${ }^{2,3}$ The most common organs affected by hydatid cyst are the liver (70\%) and lungs (25\%). However, spleen, kidneys, bile ducts, mesentery, heart, brain, and musculoskeletal or soft tissue may also be affected. ${ }^{4}$ Uncommon locations of hydatid cysts include the peritoneal cavity (10-16\%), spleen (3\%), brain (3\%), musculoskeletal system (0.5-4\%), heart (2\%), kidney (1.5-4\%), retroperitoneum (1\%), and supraclavicular region. ${ }^{5}$

The larval stage, also known as the metacestode is found in the small intestine of dogs. Humans get infected by ingestion of eggs of Echinococcus. They serve as the accidental intermediate host in whom the life-cycle of the Echinococcus reaches a dead end. The parasite eggs hatch in the small intestine of humans. From there, they pass into the portal venous system or lymphatic system and reach the liver and lungs. They also enter the systemic circulation after crossing the hepatic sinusoids or pulmonary capillary barriers and thus affect all parts of the body. ${ }^{6}$ Our case was unique because the patient suffered from a hydatid cyst in the subcutaneous plane of the supraclavicular region with no involvement of liver and lungs.

FNAC is not considered a diagnostic tool for hydatid cyst and is discouraged to be used as such. ${ }^{7}$ Diagnostic modalities include imaging studies like ultra sonogram, CT and MRI. Imaging studies are sensitive (more than $90 \%$ for USG and CT scan) diagnostic tools. ${ }^{5}$ Other diagnostic studies include ELISA for Echinococcal antibody. Serology is 80 to $100 \%$ sensitive and 88 to $96 \%$ specific for liver cyst infection but less sensitive for lung (50 to 56\%) or other organ ( 25 to $56 \%$ ) involvement. ${ }^{8}$ In our patient, both the imaging studies and serology were negative and non-contributory for the diagnosis of hydatid disease.

Surgical excision of the cyst (pericystectomy) is considered the treatment of choice. PAIR (puncture, aspiration, injection and re-aspiration) is a safe and effective alternative to surgery, and has partly replaced surgery as the treatment of choice. ${ }^{9}$ Anthelmintic chemotherapy (albendazole preferred to mebendazole) has shown improvement in most patients, albeit a very small $(29 \%)$ cure rate. ${ }^{8}$ Currently, chemotherapy has been restricted to pre and post operative use to prevent recurrence and as sole therapy for inoperable cases.

In conclusion, hydatid cyst disease can present in a rare form. Cystic echinococcosis should be considered in the differential diagnosis of $r$ a cystic swelling in any body part.

\section{References:}

1. Arora V, Nijjar IS, Gill KS, Singh G. Primary hydatid cyst of muscle-A rare site. Indian J Radiol Imaging. 2006; 16:239-41. doi: http://dx.doi.org/10.4103/09713026.29100

2. Safioleas MC, Misiakos EP, Kouvaraki M, et al. Hydatid disease of the liver. A continuing surgical problem. Arch Surg 2006; 141: 1101-8

doi: http://dx.doi.org/10.1001/archsurg.141.11.1101 
3. Ashok KB: Old disease... New location...Surgeons be alerted. International Journal of Collaborative Research on Internal Medicine \& Public Health; 2011; 3(4): 328-32 No doi

4. Ahmad S, Jalil S, Saleem Y, et al. Hydatid cysts at unusual sites: Reports of two cases in the neck and breast. J Pak Med Assoc. 2010; 60:232-4 No doi

5. .Gole SG, Gole GN, Satyanarayana V. Unusual presentation of hydatid cyst: A case series with review of literature. The Internet Journal of Parasitic Diseases. 2013; 6 (1): . doi: $10.5580 / 2 c e 2$

6. Iynen I, Sogut O, Guldur ME, et al. Primary hydatid cyst: An unusual cause of a mass in the supraclavicular region of the neck. J Clin Med Res. 2011; 3:52-4 No doi

7. Chakrabarti I, Goswami BK. Primary hydatid cyst of the neck diagnosed by aspiration cytology. Tropical Parasitology. 2012;2(2):127-128. doi:10.4103/2229-5070.105179.

8. King CH, Fairley JK: Cestodes (tapeworms), in Principles and practice of Infective diseases, $7^{\text {th }} \mathrm{ed}$, GL Mandell et al (eds). Churchill Livingstone Elsevier 2010

9. Wahlers K, Menezes CN, Wong ML, et al. Cystic echinococcosis in sub-Saharan Africa, Lancet Inf. Dis 2012; 12:871-80 doi: http://dx.doi.org/10.1016/S14733099(12)70155-X 